\title{
On multi-way metricity, minimality and diagonal planes
}

\author{
Matthijs J. Warrens
}

(C) The Author(s) 2008. This article is published with open access at Springerlink.com

\begin{abstract}
Validity of the triangle inequality and minimality, both axioms for twoway dissimilarities, ensures that a two-way dissimilarity is nonnegative and symmetric. Three-way generalizations of the triangle inequality and minimality from the literature are reviewed and it is investigated what forms of symmetry and nonnegativity are implied by the three-way axioms. A special form of three-way symmetry that can be deduced is equality of the diagonal planes of the three-dimensional cube. Furthermore, it is studied what diagonal plane equalities hold for the four-dimensional tesseract.
\end{abstract}

Keywords Diagonal plane equality · Tetrahedron inequality · Multi-way symmetry · Three-way block · Tesseract · Multi-way dissimilarity

\section{Mathematics Subject Classification (2000) 51K05}

\section{Introduction}

A basic concept in data analysis is dissimilarity. Let $E$ be a finite set that represents objects, individuals or variables. A dissimilarity $d$ on the set $E$ is a function from $E^{2}$ to $\mathbb{R}$, the set of reals, that satisfies for all $i, j \in E$ :

$$
\begin{aligned}
& \text { (a1) } \quad d_{i j} \geq 0 \quad \text { (nonnegativity) } \\
& \text { (a2) } \quad d_{i i}=0 \quad \text { (minimality) } \\
& \text { (a3) } \quad d_{i j}=d_{j i} \quad \text { (symmetry). }
\end{aligned}
$$

M. J. Warrens ( $₫)$

Psychometrics and Research Methodology Group,

Leiden University Institute for Psychological Research, Leiden University, Wassenaarseweg 52, P. O. Box 9555, 2300 RB Leiden, The Netherlands

e-mail: warrens@fsw.leidenuniv.nl 
All the dissimilarity functions occurring in this paper are defined on the set $E$. The dissimilarity $d$ may be constructed from the observed data.

A dissimilarity $d$ is called a metric if it is definite, $d_{i j}=0$ if and only if $i=j$, and satisfies

(a4) $d_{i j} \leq d_{j k}+d_{k i} \quad$ (triangle inequality)

for all $i, j, k \in E$. Definiteness is not studied in this paper. An example of $d$ that satisfies $(a 1)-(a 4)$, is the Euclidean distance between $i$ and $j$, that is, the length of the segment joining $i$ and $j$.

It is well known that the axiom system formed by $(a 1)-(a 4)$ is not a minimal system of axioms, since some of the axioms are redundant.

Theorem $1(a 2)+(a 4) \Rightarrow(a 1),(a 3)$.

Proof Supplying (a4) with triple $(i, j, j)$ we obtain $d_{i j} \leq d_{j i}$. Supplying (a4) with $(j, i, j)$ we obtain $d_{j i} \leq d_{i j}$, and hence $d_{i j}=d_{j i}$. This completes the proof of symmetry.

If $d$ is symmetric, then supplying ( $a 4)$ with triple $(i, i, j)$ proves nonnegativity.

Thus, the important axioms in system $(a 1)-(a 4)$ are minimality and the triangle inequality, since these two conditions imply nonnegativity and symmetry.

Dissimilarity models form the characteristic structure of multidimensional scaling and hierarchical cluster analysis representations. Three-way generalizations of these models have been studied by only a few authors (Heiser and Bennani 1997; Diatta 2004, 2006, 2007). Joly and Le Calvé (1995) and Bennani-Dosse (1993) consider methods for three-way cluster analysis, and Diatta (2004) considers a relation between the theory of formal concepts and multi-way clustering. Daws (1996) incorporated three-way information in the analysis of free-sorting data. Methods for multi-way multidimensional scaling can be found in Cox et al. (1991), Heiser and Bennani (1997), Gower and De Rooij (2003) and Nakayama (2005). Cox et al. (1991), Diatta (2006) and Daws (1996) convincingly showed that multi-way dissimilarities may be used to detect possible higher-order relations between the objects. However, Gower and De Rooij (2003) concluded that two-way and three-way multidimensional scaling give very similar results.

One may be interested in knowing whether there is a result analogous to Theorem 1 for a three-way or multi-way axiomatization. Deza and Rosenberg $(2000,2005)$ pointed to the fact that there is a vast literature on multi-way metrics that extend the usual two-way metric. For the three-way case, the central axioms are three-way symmetry and the tetrahedron inequality. Another axiom is the condition that the value of the three-way dissimilarity is zero if two objects are the same. Although this requirement makes perfect sense in geometry, it is too restrictive for three-way applications in statistics (Joly and Le Calvé 1995; Heiser and Bennani 1997). The weaker requirement that the value of the three-way dissimilarity is zero if all three objects are the same, is part of the three-way axiomatizations proposed in Joly and Le Calvé (1995) and Heiser and Bennani (1997). 
In this paper it is shown that different forms of nonnegativity and multi-way symmetry may be deduced from generalizations of minimality and the classical twoway triangle inequality. In the next section we consider the axioms that are central in the literature on multi-way metrics discussed in Deza and Rosenberg (2000, 2005). It is shown that three-way symmetry and nonnegativity may be deduced from the other two axioms. In section three we review an axiom system that is discussed in Heiser and Bennani (1997). For this system of three-way axioms we derive a result that is similar to Theorem 1. More precisely, it is shown that a special form of three-way symmetry, equality of the diagonal planes of the three-dimensional cube, may be deduced. In section four it is studied what diagonal plane equalities hold for the four-dimensional tesseract. Section five contains a discussion.

\section{Three-way symmetry}

A three-way dissimilarity $t$ on the set $E$ is a function from $E^{3}$ to $\mathbb{R}$. Deza and Rosenberg $(2000,2005)$ consider the following three-way extensions of $(a 1)-(a 4)$ :
(b1) $t_{i j k} \geq 0$
(nonnegativity)
(b2) $t_{i i j}=t_{i j i}=t_{j i i}=0$
(b3) $t_{i j k}=t_{i k j}=t_{j i k}=t_{j k i}=t_{k i j}=t_{k j i}$
(symmetry)
(b4) $t_{i j k} \leq t_{j k l}+t_{k l i}+t_{l i j}$
(tetrahedron inequality)

for all $i, j, k, l \in E$. The axiom (b3) captures the fact that the value of $t_{i j k}$ is independent of the order of $i, j$ and $k$. Interpreting $t_{i j k}$ as the area of the triangle with vertices $i, j$ and $k,(b 4)$ means that the area of each triangle face of the tetrahedron formed by $i, j, k$ and $l$ does not exceed the sum of the areas of the remaining faces.

Example 1 The function

$$
t_{i j k}^{\mathrm{S}}=\frac{d_{i j}+d_{j k}+d_{k i}}{2}
$$

is referred to as the semi-perimeter distance in Joly and Le Calvé (1995) and BennaniDosse (1993). The function $2 t^{\mathrm{S}}$ is called the perimeter distance and is used in Heiser and Bennani (1997) and Gower and De Rooij (2003). If $d_{i j}, d_{j k}$ and $d_{k i}$ are the side lengths of the triangle with vertices $i, j$ and $k$, then function $t_{i j k}^{\mathrm{S}}$ is the semi-perimeter of the triangle.

Function $t^{\mathrm{S}}$ satisfies $(b 1)$ if $(a 1)$ is valid, and (b3) and (b4), the tetrahedron inequality, if (a3) is valid. If (a2) is valid, then $t_{i i j}^{\mathrm{S}}=t_{i j i}^{\mathrm{S}}=t_{j i i}^{\mathrm{S}}$, but not (b2) in general. Summarizing, $t^{\mathrm{S}}$ satisfies $(b 1)-(b 4)$ if $d$ satisfies $(a 1)-(a 3)$, that is, $d$ must be a dissimilarity, not necessarily a metric.

Example 2 Heron's formula states that in Euclidean space the area of a triangle whose sides have lengths $d_{i j}, d_{j k}$ and $d_{k i}$ is

$$
t_{i j k}^{\mathrm{A}}=\sqrt{t_{i j k}^{\mathrm{S}}\left(t_{i j k}^{\mathrm{S}}-d_{i j}\right)\left(t_{i j k}^{\mathrm{S}}-d_{j k}\right)\left(t_{i j k}^{\mathrm{S}}-d_{k i}\right)}
$$


where $t_{i j k}^{\mathrm{S}}$ is the semi-perimeter of the triangle. Since $d$ is the Euclidean distance, function $t_{i j k}^{\mathrm{A}}$ satisfies $(b 1)-(b 4)$.

Similar to the two-way case, some of the axioms of system $(b 1)-(b 4)$ are redundant.

Theorem $2(b 2)+(b 4) \Rightarrow(b 1),(b 3)$.

Proof Supplying (b4) with quadruples $(i, j, k, i),(j, k, i, j)$ and $(k, i, j, k)$, we obtain, respectively, $t_{i j k} \leq t_{j k i}, t_{j k i} \leq t_{k i j}$ and $t_{k i j} \leq t_{i j k}$, which leads to $t_{i j k}=t_{j k i}=t_{k i j}$. We also have $t_{j i k}=t_{i k j}=t_{k j i}$. Furthermore, supplying $(b 4)$ with $(i, j, k, j)$ and $(k, j, i, j)$, we obtain, respectively, $t_{i j k} \leq t_{k j i}$ and $t_{k j i} \leq t_{i j k}$. Hence, $t_{i j k}=t_{k j i}$. This completes the proof of symmetry.

If $t$ is symmetric, then supplying (b4) with quadruple $(i, i, j, k)$ proves $(b 1)$.

Thus, Theorem 2 is a result that is analogous to Theorem 1 . The important axioms in system (b1)-(b4) are (b2) and (b4), the tetrahedron inequality, since these two conditions imply nonnegativity and symmetry. Moreover, the formulation of a multi-way axiomatization, for which a result analogous to Theorem 2 holds, is straightforward.

\section{Diagonal planes of the cube}

Joly and Le Calvé (1995) and Heiser and Bennani (1997) have proposed three-way extensions of ( $a 2)$ and $(a 4)$ that are different from (b2) and (b4). Deza and Rosenberg $(2000,2005)$ found $(b 2)$ too restrictive and dropped the axiom entirely. We consider the following three-way conditions:

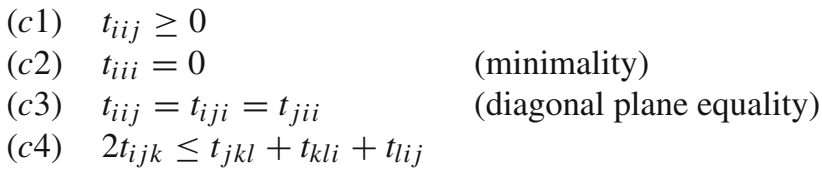

for all $i, j, k, l \in E$. Condition ( $c 1)$ is weaker than ( $b 1)$. Instead of using ( $b 2)$, both Joly and Le Calvé (1995) and Heiser and Bennani (1997) proposed the weaker requirement $(c 2)$ as the three-way generalization of minimality. Although ( $c 2)$ is a more realistic requirement in three-way data analysis, the condition is not strong enough to derive symmetry, using either (b4) or even (c4), as in Theorem 2. Both Heiser and Bennani (1997) and Joly and Le Calvé (1995) simply assume that the three-way distances satisfy (b3). In addition, the latter authors require that $t_{i j k} \geq t_{i i k}$, which together with (c2), implies (b1).

Condition (c3) is a weaker condition than three-way symmetry (b3). Let $\left\{t_{i i j}\right\}$, $\left\{t_{i j i}\right\}$ and $\left\{t_{j i i}\right\}$ be the three matrices that are formed by cutting the cube diagonally, starting at one of the three edges joining at the corner $t_{111}$ (Fig. 1). Condition (c3) requires that the three matrices are equal.

There are multiple ways for introducing three-way metricity. Deza and Rosenberg (2000, 2005) consider (b4), the tetrahedron inequality. Joly and Le Calvé (1995) proposed the inequality $t_{i j k} \leq t_{j k l}+t_{i k l}$, whereas Heiser and Bennani (1997) proposed 

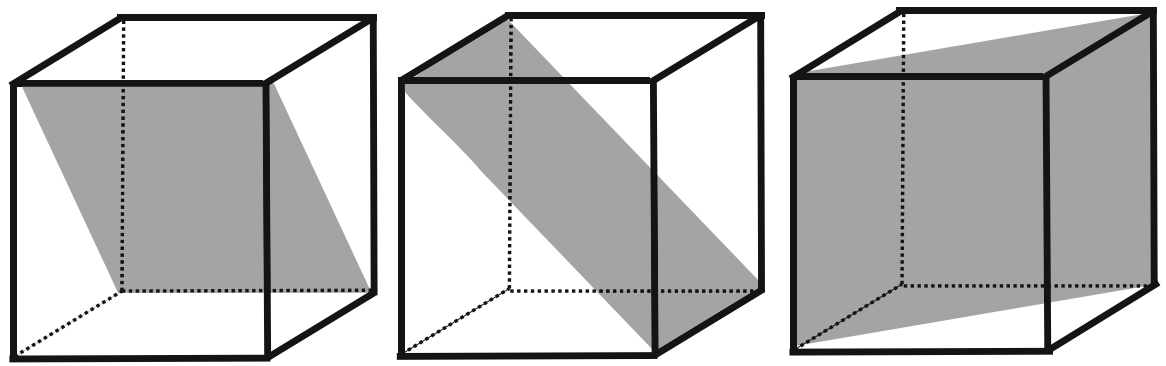

Fig. 1 Diagonal planes that are formed by cutting the cube diagonally, starting at one of the three edges joining at the vertex $t_{111}$

(c4) as a generalization of the triangle inequality (a4). Inequality (c4) is a variant of (b4) which gives twice as much weight to the left-hand side of the inequality. We have $(c 4) \Rightarrow(b 4)$. It should be noted that (c4) is actually a variant of the inequality in Heiser and Bennani (1997, p. 191), which is given by $2 t_{i j k} \leq t_{i k l}+t_{j k l}+t_{i j l}$. The rearrangement of the indices in $(c 4)$ is required to obtain Theorem 3.

Example 3 Let $i, j$ and $k$ be three binary (0/1) vectors of length $n$. Let $n_{i j k}^{111}$ and $n_{i j k}^{000}$ denote respectively the number of $1 \mathrm{~s}$ and 0 s that the three objects share in the same positions. Cox et al. (1991, p. 200) and Heiser and Bennani (1997, p. 196) define the three-way Jaccard dissimilarity as

$$
t_{i j k}^{\mathrm{Jac}}=1-\frac{n_{i j k}^{111}}{n-n_{i j k}^{000}} .
$$

In words, $t_{i j k}^{\mathrm{Jac}}$ is equal to one minus the number of $1 \mathrm{~s}$ that $i, j$ and $k$ have in the same positions, divided by the number of positions were at least one 1 occurs.

It is readily verified that $t^{\mathrm{Jac}}$ satisfies $(b 1),(b 3),(c 1)$ and (c3). Heiser and Bennani (1997, p. 196) showed that $t^{\mathrm{Jac}}$ also satisfies $(c 4)$.

Conditions (c2) and (c4) do not imply (b1) and (b3). Instead we have the following result.

Theorem $3(c 2)+(c 4) \Rightarrow(c 1),(c 3)$.

In the proof of Theorem 3 we use the following lemma.

Lemma 1 Let $a, b$ and $c$ be real numbers. If $2 a \leq b+c, 2 b \leq a+c$ and $2 c \leq a+b$, then $a=b=c$.

Remark The following proof was provided by an anonymous referee. The proof is more elegant than the proof originally provided by the author.

Proof Adding the first two inequalities we obtain $2 a+2 b \leq a+b+2 c$, that is, $a+b \leq 2 c$. The third inequality is $2 c \leq a+b$, so we must have $a+b=2 c$ and $a+b+c=3 c$. Equality then follows from $a+b+c=3 a=3 b=3 c$. 
Proof of Theorem 3 Supplying (c4) with quadruples $(j, i, i, i),(i, j, i, i)$ and $(i, i, j, i)$, we obtain, respectively, $2 t_{j i i} \leq t_{i i j}+t_{i j i}, 2 t_{i j i} \leq t_{j i i}+t_{i i j}$ and $2 t_{i i j} \leq$ $t_{i j i}+t_{j i i}$. Condition (c3) then follows from application of Lemma 1.

If $(c 3)$ holds, then supplying $(c 4)$ with quadruple $(i, i, i, j)$ proves $(c 1)$.

If (c3) is valid, then we have $t_{i i j}=t_{i j i}=t_{j i i}$ and $t_{i j j}=t_{j i j}=t_{j j i}$, but not $t_{i i j}=t_{j j i}$, that is, the diagonal planes are not necessarily symmetric. Heiser and Bennani (1997) and Joly and Le Calvé (1995) therefore use the additional requirement $t_{i i j}=t_{i j j}$ (which is referred to as the "diagonal-plane equality" in Heiser and Bennani (1997)). Supplying (c4) with quadruple $(i, j, i, j)$ we obtain $t_{i j i} \leq 2 t_{j i j}$. If $(c 2)$ and (c4) are valid, $t_{i i j} \neq t_{i j j}$, but $t_{i i j}$ is bounded from above by $2 t_{i j j}$ (and vice versa).

\section{Diagonal planes of the tesseract}

In this section we investigate the relationships between various forms of four-way metricity and definitions of minimality, and diagonal planes of the four-dimensional tesseract. We consider two axiom systems for four-way distances. The four-way dissimilarity $q$ on the set $E$ is a function from $E^{4}$ to $\mathbb{R}$. The four-way generalizations of $(c 1)-(c 4)$ are given by:

$$
\begin{array}{ll}
(d 1) & q_{i i i j} \geq 0 \\
(d 2) & q_{i i i i}=0 \\
(d 3) & q_{i i i j}=q_{i i j i}=q_{i j i i}=q_{j i i i} \\
(d 4) & 3 q_{i j k l} \leq q_{j k l m}+q_{k l m i}+q_{l m i j}+q_{m i j k}
\end{array}
$$

for all $i, j, k, l, m \in E$. Up to three objects may be identical with regard to system $(d 1)-(d 4)$. Similar to $(c 3)$, condition $(d 3)$ requires that the four diagonal planes that are formed by cutting the tesseract diagonally, starting at one of the four edges joining at the vertex $q_{1111}$, are equal. Figure 2 consists of four three-way projections of the four-dimensional tesseract. The figure provides a visual impression of the four planes considered in (c3). Condition ( $d 4)$ is the four-way generalization of $c 4$ and $a 4$.

Example 4 Let $i, j, k$ and $l$ be four binary (0/1) vectors of length $n$. Let $n_{i j k l}^{1111}$ and $n_{i j k l}^{0000}$ denote respectively the number of 1 s and 0 s that the four objects share in the same positions. Cox et al. (1991, p. 200) define the four-way Jaccard dissimilarity as

$$
q_{i j k l}^{\mathrm{Jac}}=1-\frac{n_{i j k l}^{1111}}{n-n_{i j k l}^{0000}} .
$$

In words, $q_{i j k l}^{\mathrm{Jac}}$ is one minus the number of $1 \mathrm{~s}$ that $i, j, k$ and $l$ share in the same positions, divided by the number of positions were at least one 1 occurs. It is readily verified that $q^{\mathrm{Jac}}$ satisfies $(d 1),(d 2)$ and $(d 3)$. Theorem 4 is used to show that $q^{\mathrm{Jac}}$ also satisfies $(d 4)$.

Theorem 4 The function $q^{J a c}$ satisfies axiom $(d 4)$. 

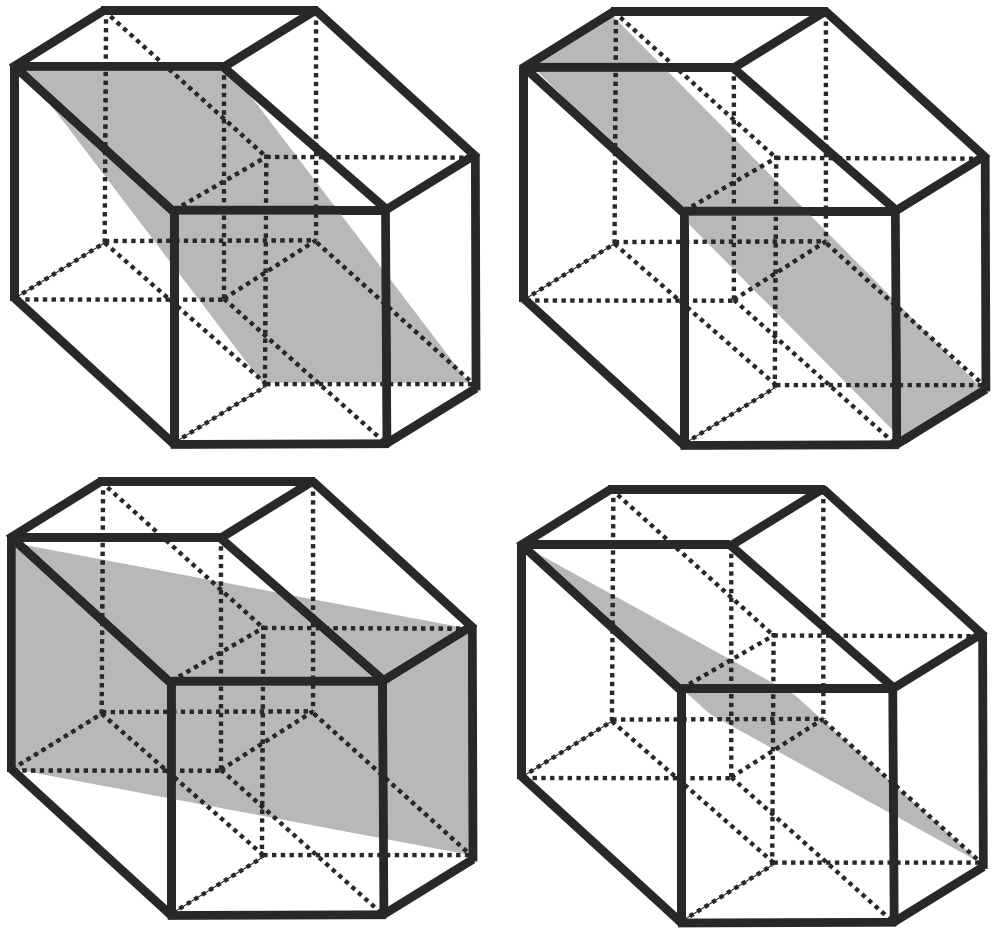

Fig. 2 Diagonal planes that are formed by cutting the three-way projection of the four-dimensional tesseract diagonally, starting at one of the four edges joining at the vertex $q_{111}$

Proof Let $i, j, k, l$ and $m$ be five binary vectors of length $n$. Let the $n \times 5$ matrix $\mathbf{X}$ consists of the five column vectors positioned next to each other. Each row of $\mathbf{X}$ is a score pattern of $0 \mathrm{~s}$ and $1 \mathrm{~s}$. The maximum number of different score patterns is equal to $2^{5}=32$. Let $n_{i j k l m}^{11010}$ denotes the number of rows of $\mathbf{X}$ were a 1 occurs for vectors (columns) $i, j$ and $l$, and a 0 for vectors $k$ and $m$. The total number of rows of $\mathbf{X}, n$, can be decomposed into $n=n_{i j k l m}^{11111}+n_{i j k l m}^{11110}+n_{i j k l m}^{11101}+\cdots+n_{i j k l m}^{00001}+n_{i j k l m}^{00000}$. The right-hand side of this equality has 32 components.

We have the inequality

$$
\begin{aligned}
n \geq & n_{i j k l m}^{11111}+n_{i j k l m}^{11110}+n_{i j k l m}^{11101}+n_{i j k l m}^{11011}+n_{i j k l m}^{10111}+n_{i j k l m}^{01111} \\
& +n_{i j k l m}^{10000}+n_{i j k l m}^{01000}+n_{i j k l m}^{00100}+n_{i j k l m}^{00010}+n_{i j k l m}^{00001}+n_{i j k l m}^{00000} .
\end{aligned}
$$

Adding $3\left(n_{i j k l m}^{11111}+n_{i j k l m}^{11110}+n_{i j k l m}^{00001}+n_{i j k l m}^{00000}\right)$ to both sides of (1), we obtain

$$
\begin{aligned}
n+ & 3 n_{i j k l m}^{11111}+3 n_{i j k l m}^{11110}+3 n_{i j k l m}^{00001}+3 n_{i j k l m}^{00000} \\
\geq & 4 n_{i j k l m}^{11111}+4 n_{i j k l m}^{11110}+n_{i j k l m}^{11101}+n_{i j k l m}^{11011}+n_{i j k l m}^{10111}+n_{i j k l m}^{01111} \\
& +n_{i j k l m}^{10000}+n_{i j k l m}^{01000}+n_{i j k l m}^{00100}+n_{i j k l m}^{00010}+4 n_{i j k l m}^{00001}+4 n_{i j k l m}^{00000} .
\end{aligned}
$$


Using the five identities

$$
\begin{aligned}
n_{i j k l}^{1111}+n_{i j k l}^{0000} & =n_{i j k l m}^{11111}+n_{i j k l m}^{11110}+n_{i j k l m}^{00000}+n_{i j k l m}^{00001}, \\
n_{i j k m}^{1111}+n_{i j k m}^{0000} & =n_{i j k l m}^{1111}+n_{i j k l m}^{11101}+n_{i j k l m}^{00000}+n_{i j k l m}^{00010}, \\
n_{i j l m}^{1111}+n_{i j l m}^{0000} & =n_{i j k l m}^{11111}+n_{i j k l m}^{11011}+n_{i j k l m}^{00000}+n_{i j k l m}^{00100}, \\
n_{i k l m}^{1111}+n_{i k l m}^{0000} & =n_{i j k l m}^{11111}+n_{i j k l m}^{10111}+n_{i j k l m}^{00000}+n_{i j k l m}^{01000}, \\
n_{j k l m}^{1111}+n_{j k l m}^{0000} & =n_{i j k l m}^{11111}+n_{i j k l m}^{01111}+n_{i j k l m}^{00000}+n_{i j k l m}^{10000},
\end{aligned}
$$

inequality (2) can be written as

$$
\begin{aligned}
& \left(n-n_{i j k m}^{1111}-n_{i j k m}^{0000}\right)+\left(n-n_{i j l m}^{1111}-n_{i j l m}^{0000}\right) \\
& \quad+\left(n-n_{i k l m}^{1111}-n_{i k l m}^{0000}\right)+\left(n-n_{j k l m}^{1111}-n_{j k l m}^{0000}\right)-3\left(n-n_{i j k l}^{1111}-n_{i j k l}^{0000}\right) \\
& \quad \geq 4 n_{i j k l m}^{11110}+4 n_{i j k l m}^{00001} .
\end{aligned}
$$

Using the five variants of identity

$$
\begin{aligned}
\left(n-n_{i j k l}^{111}-n_{i j k l}^{0000}\right) & =\left(n-n_{i j k l}^{0000}\right) q_{i j k l}^{\mathrm{Jac}} \\
& =\left(n-n_{i j k l m}^{00000}\right) q_{i j k l}^{\mathrm{Jac}}-n_{i j k l m}^{00001} q_{i j k l}^{\mathrm{Jac}}
\end{aligned}
$$

in (3), we obtain

$$
\begin{aligned}
(n & \left.-n_{i j k l m}^{00000}\right)\left(q_{i j k m}^{\mathrm{Jac}}+q_{i j l m}^{\mathrm{Jac}}+q_{i k l m}^{\mathrm{Jac}}+q_{j k l m}^{\mathrm{Jac}}-3 q_{i j k l}^{\mathrm{Jac}}\right) \\
\geq & 4 n_{i j k l m}^{11110}+n_{i j k l m}^{00001}\left(4-3 q_{i j k l}^{\mathrm{Jac}}\right) \\
& +n_{i j k l m}^{00010} q_{i j k m}^{\mathrm{Jac}}+n_{i j k l m}^{00100} q_{i j l m}^{\mathrm{Jac}}+n_{i j k l m}^{01000} q_{i k l m}^{\mathrm{Jac}}+n_{i j k l m}^{10000} q_{j k l m}^{\mathrm{Jac}} .
\end{aligned}
$$

Since $\left(n-n_{i j k l m}^{00000}\right) \geq 0$ and $q_{i j k l}^{\mathrm{Jac}} \leq 1$, we conclude that $q^{\mathrm{Jac}}$ satisfies $(d 4)$.

For conditions $(d 1)-(d 4)$ we have the following result.

Theorem $5(d 2)+(d 4) \Rightarrow(d 1),(d 3)$.

In the proof of Theorem 5 we use the following lemma.

Lemma 2 Let $a, b, c$ and $d$ be real numbers. If $3 a \leq b+c+d, 3 b \leq a+c+d$, $3 c \leq a+b+d$ and $3 d \leq a+b+c$, then $a=b=c=d$.

Proof Adding the first three inequalities we obtain $3 a+3 b+3 c \leq 2 a+2 b+2 c+3 d$, that is, $a+b+c \leq 3 d$. The third inequality is $3 d \leq a+b+c$, so we must have $a+b+c=3 d$ and $a+b+c+d=4 d$. Equality then follows from $a+b+c+d=$ $4 a=4 b=4 c=4 d$. 

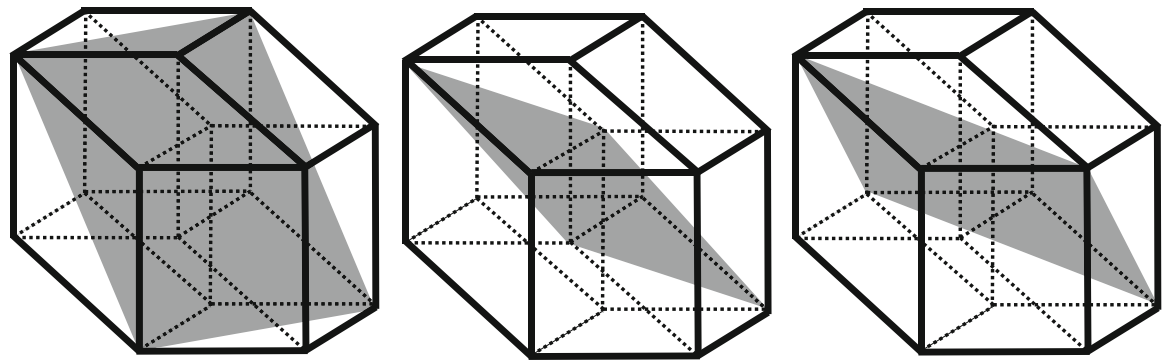

Fig. 3 Three diagonal planes of the three-way projection of the four-dimensional tesseract. The diagonal planes are different from the ones considered in Fig. 2

Proof of Theorem 5 Supplying (d4) with tuples $(j, i, i, i, i),(i, j, i, i, i),(i, i, j, i, i)$ and $(i, i, i, j, i)$, we obtain, respectively, $3 q_{j i i i} \leq q_{i i i j}+q_{i i j i}+q_{i j i i}, 3 q_{i j i i} \leq q_{j i i i}+$ $q_{i i i j}+q_{i i j i}, 3 q_{i i j i} \leq q_{i j i i}+q_{j i i i}+q_{i i i j}$ and $3 q_{i i i j} \leq q_{i i j i}+q_{i j i i}+q_{j i i i}$. Condition (d3) then follows from application of Lemma 2.

If $(d 3)$ holds, then supplying $(d 4)$ with tuple $(i, i, i, i, j)$ proves $(d 1)$.

Note that validity of $(d 3)$ does not ensure that the diagonal planes in Fig. 2 are symmetric. Similar to the three-way case, symmetry of these planes requires an additional requirement. Theorem 5 is a result analogous to Theorem 3 for four-way axioms $(d 1)-(d 4)$. Moreover, the formulation of a multi-way axiomatization, for which a result analogous to Theorem 5 holds, is straightforward.

We consider an additional axiomatization for the four-way case:

$$
\begin{aligned}
& \text { (e1) } \quad q_{i i j j} \geq 0 \\
& (e 2) \quad q_{i i i j}=q_{i i j i}=q_{i j i i}=q_{j i i i}=0 \\
& (e 3) \quad q_{i i j j}=q_{i j i j}=q_{i j j i}=q_{j i i j}=q_{j i j i}=q_{j j i i} \\
& (e 4) \quad 2 q_{i j k l} \leq q_{j k l m}+q_{k l m i}+q_{l m i j}+q_{m i j k}
\end{aligned}
$$

for all $i, j, k, l \in E$. Condition ( $e 2)$ is a stronger requirement than $(d 2)$ and $(d 3)$, but $(e 4) \Leftarrow(d 4)$. Unlike $(d 3)$, condition $(e 3)$ is a diagonal plane equality that does not involve the elements of the edges joining at the corner $q_{1111}$. Figure 3 consists of three three-way projections of the four-dimensional tesseract. The figure provides, similar to Fig. 2, a visual impression of the three diagonal planes that are considered in $(e 3)$ : $\left\{q_{i i j j}\right\},\left\{q_{i j i j}\right\}$ and $\left\{q_{i j j i}\right\}$. Condition (e3) does not only require the three matrices to be equal, they must be symmetric as well.

Example 5 A function that extends Example 1, is the four-way perimeter model

$$
q_{i j k l}^{\mathrm{P}}=d_{i j}+d_{j k}+d_{k l}+d_{l i}+d_{i k}+d_{j l}
$$

Function $q^{\mathrm{P}}$ is the sum of the six two-way dissimilarities that can be formed given a group of four objects. Function $q^{\mathrm{P}}$ satisfies $(e 1)$ if $(a 1)$ is valid, and (e3) and (e4) if $(a 3)$ is valid. If (a2) and (a3) are valid, then $q^{\mathrm{P}}$ satisfies $(d 3)$, but not (e2) in general.

We have the following result for system $(e 1)-(e 4)$. 
Theorem $6(e 2)+(e 4) \Rightarrow(e 1),(e 3)$.

Proof Supplying (e4) with tuples $(j, j, i, i, i),(i, j, j, i, i)$ and $(i, i, j, j, i)$, we obtain, respectively, $2 q_{j j i i} \leq q_{i i j j}+q_{i j j i}, 2 q_{i j j i} \leq q_{j j i i}+q_{i i j j}$ and $2 q_{i i j j} \leq q_{i j j i}+q_{j j i i}$. By Lemma $1, q_{i i j j}=q_{i j j i}=q_{j j i i}$. Supplying $(e 4)$ with tuples $(j, i, j, i, i),(j, i, i, j, i)$, and $(i, j, i, j, i)$, we obtain, respectively, $2 q_{j i j i} \leq q_{j i i j}+q_{i j i j}, 2 q_{j i i j} \leq q_{i j i j}+q_{j i j i}$ and $2 q_{i j i j} \leq q_{j i j i}+q_{j i i j}$. By Lemma $1, q_{i j i j}=q_{j i i j}=q_{j i j i}$. We also have $q_{j i j i}=q_{i j j i}=q_{i j i j}$. Condition (e3) then follows from combining the three results.

If $(e 3)$ holds, then supplying $(e 4)$ with tuple $(i, i, i, j, j)$ proves $(e 1)$.

\section{Discussion}

A two-way function is a dissimilarity if it satisfies the axioms of nonnegativity, minimality and symmetry. A dissimilarity is called a metric if it is definite and if it satisfies the triangle inequality. A well-known result is that validity of both the triangle inequality and minimality ensures that the two-way dissimilarity is nonnegative and symmetric. In this paper we reviewed three-way generalizations and formulated four-way generalizations of the two-way axioms. We derived results for three-way and four-way dissimilarities that are similar to the well-known result for two-way dissimilarities.

To deduce multi-way or total symmetry, it is required that the dissimilarity has zero value in the case that two objects are identical. This is a rather strong requirement. Using weaker versions of minimality, combined with different forms of metricity, we derived various diagonal plane equalities for three-way and four-way dissimilarities. There are multiple ways for introducing multi-way metricity. Examples 1 to 5 demonstrate that for each multi-way metric inequality that is considered in this paper, there is at least one function that satisfies the inequality.

Condition (c4) is a strong generalization of the triangle inequality proposed in Heiser and Bennani (1997). Examples of functions that satisfy inequality $(c 4)$ can be found in Joly and Le Calvé (1995), Bennani-Dosse (1993), Heiser and Bennani (1997) and Nakayama (2005). However, it should be noted that, although there are many cases in which this three-way metric inequality is valid, the three-way models can be used regardless of the validity of the three-way axiom. For example, the three-way multidimensional scaling proposed in Gower and De Rooij (2003) merely requires that the underlying two-way dissimilarities satisfy the triangle inequality, since the scaling method uses three-way dissimilarities that are linear transformations of the two-way dissimilarities. The results on dependencies between various axioms derived in this paper are therefore of theoretical interest only.

For the four-way, five-way and general multi-way polytope it should be possible to deduce equality of not only planes, but also higher-dimensional manifolds like cubes and tesseracts, under specific conditions. For example, using $(e 2)$ and supplying (e4) with tuples $(j, k, i, i, i),(i, j, k, i, i)$ and $(i, i, j, k, i)$, we obtain, respectively, $2 q_{j k i i} \leq q_{i i j k}+q_{i j k i}, 2 q_{i j k i} \leq q_{j k i i}+q_{i i j k}$ and $2 q_{i i j k} \leq q_{i j k i}+q_{j k i i}$. By Lemma 1 , $q_{i i j k}=q_{i j k i}=q_{j k i i}$. Using similar arguments we may obtain $q_{i j i k}=q_{j i k i}=q_{k i i j}$. Clearly, multi-way distances generate a lot of new possibilities and properties that may be studied. 
Acknowledgments The author thanks Hans-Hermann Bock and three anonymous reviewers for their helpful comments and valuable suggestions on earlier versions of this article.

Open Access This article is distributed under the terms of the Creative Commons Attribution Noncommercial License which permits any noncommercial use, distribution, and reproduction in any medium, provided the original author(s) and source are credited.

\section{References}

Bennani-Dosse M (1993) Analyses Métriques á Trois Voies. Unpublished PhD thesis, Université de Haute Bretagne Rennes II, France

Cox TF, Cox MAA, Branco JA (1991) Multidimensional scaling for $n$-tuples. Br J Math Stat Psychol 44:195-206

Daws JT (1996) The analysis of free-sorting data: Beyond pairwise cooccurrences. J Classif 13:57-80

Deza M-M, Rosenberg IG (2000) n-Semimetrics. Eur J Comb Spec Issue Discrete Metric Spaces 21:797806

Deza M-M, Rosenberg IG (2005) Small cones of $m$-hemimetrics. Discrete Math 291:81-97

Diatta J (2004) A relation between the theory of formal concepts and multiway clustering. Pattern Recogn Lett 25:1183-1189

Diatta J (2006) Description-meet compatible multiway dissimilarities. Discrete Appl Math 154:493-507

Diatta J (2007) Galois closed entity sets and $k$-balls of quasi-ultrametric multi-way dissimilarities. Adv Data Anal Classif 1:53-65

Gower JC, De Rooij M (2003) A comparison of the multidimensional scaling of triadic and dyadic distances. J Classif 20:115-136

Heiser WJ, Bennani M (1997) Triadic distance models: axiomatization and least squares representation. J Math Psychol 41:189-206

Joly S, Le Calvé G (1995) Three-way distances. J Classif 12:191-205

Nakayama A (2005) A multidimensional scaling model for three-way data analysis. Behaviormetrika 32:95-110 